Natl. Acad. Sci. U. S. A. 101:6698-6703.

6. Wagers, S.S., et al. 2004. Extravascular fibrin, plasminogen activator, plasminogen activator inhibitors, and airway hyperresponsiveness. J. Clin. Invest. 114:104-111. doi:10.1172/JCI200419569.

7. Jarjour, N.N., and Enhorning, G. 1999. Antigeninduced airway inflammation in atopic subjects generates dysfunction of pulmonary surfactant. Am. J. Respir. Crit. Care Med. 160:336-341.

8. Yager, D., Kamm, R.D., and Drazen, J.M. 1995 Airway wall liquid: sources and role as an amplifier of bronchoconstriction. Chest. 107(3 Suppl.):105S-110S.

9. Taylor, F.B., Jr., and Abrams, M.E. 1966. Effect of surface active lipoprotein on clotting and fibrinolysis, and of fibrinogen on surface tension of surface active lipoprotein. Am. J. Med. 40:346-350.

10. Putz, G., Goerke, J., Taeusch, H.W., and Clements, J.A. 1994. Comparison of captive and pulsating bubble surfactometers with the use of lung surfactants. J. Appl. Physiol. 76:1425-1431.

11. Seeger, W., Stohr, G., Wolf, H.R., and Neuhof, H. 1985. Alteration of surfactant function due to protein leakage: special interaction with fibrin monomer. J. Appl. Physiol. 58:326-338.

12. Schmiedl, A., et al. 2003. Increase of inactive intraalveolar surfactant subtypes in lungs of asthmatic brown Norway rats. Virchows Arch. 442:56-65.

13. Mead, J., Takishima, T., and Leith, D. 1970. Stress distribution in lungs: a model of pulmonary elasticity. J. Appl. Physiol. 28:596-608.

14. Kamm, R.D., and Schroter, R.C. 1989. Is airway closure caused by a liquid film instability? Respir. Physiol. 75:141-156.

15. Bayley, T., Clements, J.A., and Osbahr, A.J. 1967. Pulmonary and circulatory effects of fibrinopeptides. Circ. Res. 21:469-485.

16. Coughlin, S.R. 2000. Thrombin signaling and protease-activated receptors. Nature. 407:258-264.

17. Elias, J.A., et al. 2003. New insights into the pathogenesis of asthma. J. Clin. Invest. 111:291-297.
doi:10.1172/JCI200317748

18. Yuda, H., et al. 2004. Activated protein C inhibits bronchial hyperresponsiveness and Th2 cytokine expression in mice. Blood. 103:2196-2204.

19. Hays, S.R., and Fahy, J.V. 2003. The role of mucus in fatal asthma. Am. J. Med. 115:68-69.

20. Hoshino, M., Takahashi, M., and Aoike, N. 2001. Expression of vascular endothelial growth factor, basic fibroblast growth factor, and angiogenin immunoreactivity in asthmatic airways and its relationship to angiogenesis. J. Allergy Clin. Immunol. 107:295-301.

21. Babu, K.S., et al. 2003. Inhaled synthetic surfactant abolishes the early allergen-induced response in asthma. Eur. Respir. J. 21:1046-1049.

22. Cho, S.H., et al. 2001. Possible role of the $4 \mathrm{G} / 5 \mathrm{G}$ polymorphism of the plasminogen activator inhibitor 1 gene in the development of asthma. J. Allergy Clin. Immunol. 108:212-214.

23. Gabazza, E.C., et al. 1999. Thrombin in the airways of asthmatic patients. Lung. 177:253-262.

\title{
Apoptotic mechanisms in Alzheimer neurofibrillary degeneration: cause or effect?
}

Dennis W. Dickson

Departments of Pathology and Neuroscience, Mayo Clinic College of Medicine, Jacksonville, Florida, USA.

\begin{abstract}
Increasing evidence suggests that selective neuronal loss in neurodegenerative diseases involves activation of cysteine aspartyl proteases (caspases), which initiate and execute apoptosis. In Alzheimer disease both extracellular amyloid deposits and intracellular amyloid $\beta$ protein may activate caspases, leading to cleavage of nuclear and cytoskeletal proteins, including tau protein. Proteolysis of tau may be critical to neurofibrillary degeneration, which correlates with dementia (see the related article beginning on page 121).
\end{abstract}

Alzheimer disease (AD), the most common cause of dementia in the elderly, is associated with senile plaques and neurofibrillary tangles (NFTs), but the relationship between these two neuropathologic lesions has been difficult to discover. Senile plaques are heterogenous lesions composed of extracellular amyloid $\beta$ protein (A $\beta)$, dystrophic neuronal processes, and reactive glia (1), while NFTs are intracellular lesions composed of filamentous aggregates of the microtubuleassociated protein tau (2). Genetic factors have implicated $A \beta$ in the pathogenesis of $\mathrm{AD}$ since mutations are found in the $\mathrm{A} \beta$ precursor (APP) as well as in enzymes involved in the production of $\mathrm{A} \beta$ (reviewed

Nonstandard abbreviations used: Alzheimer disease $(\mathrm{AD})$; amyloid $\beta$ protein (A $\beta$ ); $\mathrm{A} \beta$ precursor (APP); neurofibrillary tangle (NFT); Parkinson disease (PD).

Conflict of interest: The author has declared that no conflict of interest exists.

Citation for this article: J. Clin. Invest. 114:23-27 (2004) doi:10.1172/JCI200422317. in ref. 3). Moreover, genes implicated in AD by linkage studies encode proteins that degrade $A \beta$, such as insulin-degrading enzyme. The major genetic risk factor for late-onset $\mathrm{AD}$, apolipoprotein $\mathrm{E}$, promotes $A \beta$ aggregation and colocalizes with $A \beta$ in senile plaques.

The conundrum that has plagued research on $\mathrm{AD}$ is that clinicopathologic studies have not shown strong correlations between cognitive impairment and $\mathrm{A} \beta$. In particular, the degree of cognitive impairment in AD is not as closely tied to the amount of amyloid deposited in the brain or the number of senile plaques as it is to the amount of abnormal tau protein in the brain and the density and distribution of NFTs $(4,5)$. This is not entirely surprising since NFTs are composed of proteins that are part of the neuronal cytoskeleton, which supports vital structural and dynamic neuronal functions. Amyloid, on the other hand, is a relatively innocuous extracellular deposit. It has been suggested that cytoskeletal disruption may act as the proximate cause of progressive synaptic and neuronal loss by interfering with axoplasmic and dendritic transport, which starves the cell of trophic support (6). This dysfunction and eventual death of neurons manifests clinically as cognitive impairment.

A major challenge of the amyloid cascade hypothesis for $\mathrm{AD}$ (Figure 1), which posits that amyloid formation leads to neuronal loss and dementia (7), is determining the link between $A \beta$, the protein most clearly linked to the cause of $\mathrm{AD}$, and tau, the protein that is most clearly associated with clinical manifestations of AD. The studies by Rissman and coworkers in this issue of the JCI (8) suggest that apoptotic mechanisms may be the missing link.

\section{Apoptosis in AD}

Apoptosis has been the focus of intense research in the last several decades as a means of controlling cell populations in normal development and inflammation through programmed cell death. Failure to control cell numbers through apoptosis is common in cancer, while excessive apoptosis is viewed to play a role in a number of neurologic disorders in addition to $\mathrm{AD}$, including stroke and Parkinson disease (PD) (9). 


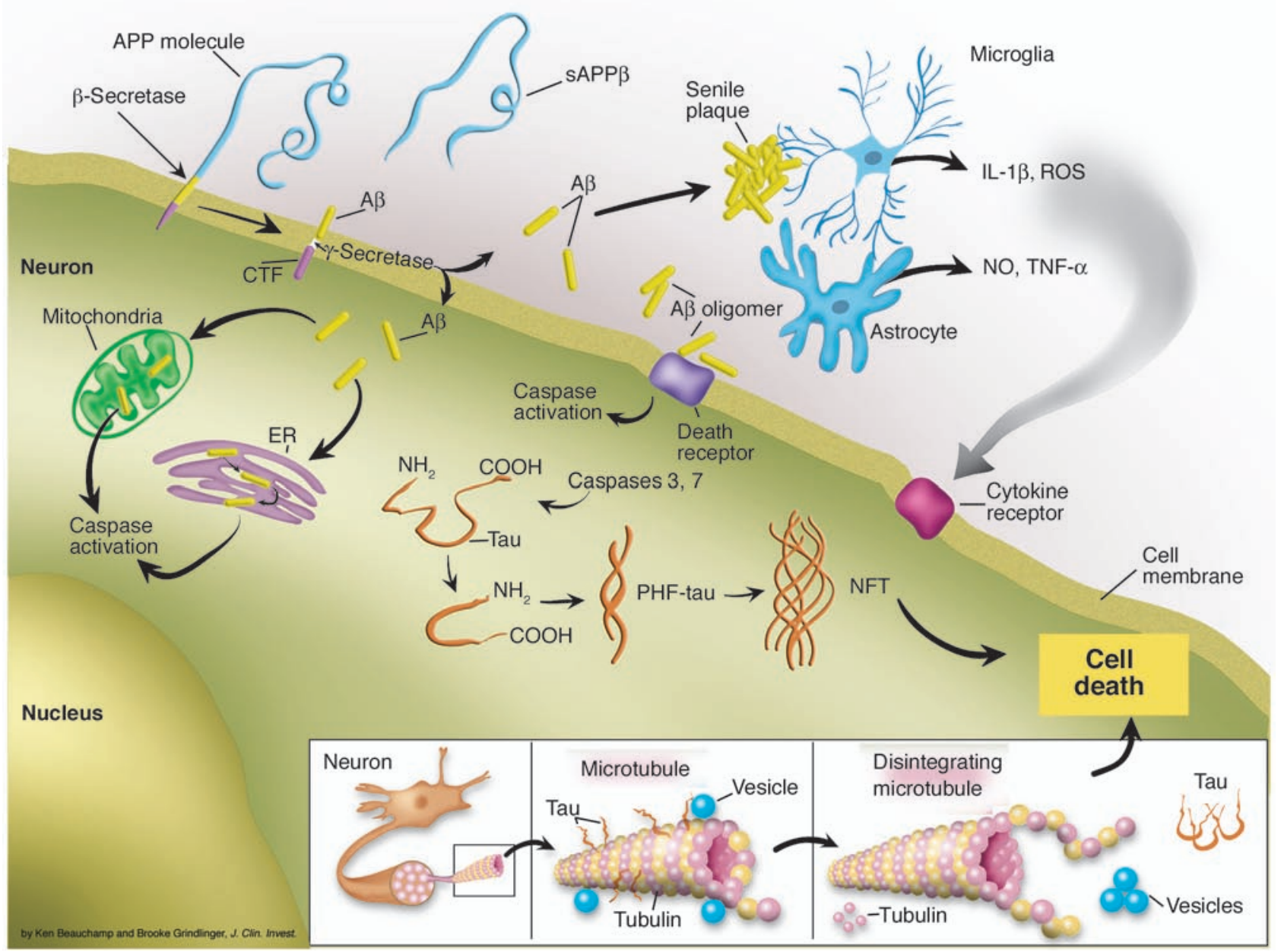

\section{Figure 1}

Proteolytic processes contribute to the amyloid cascade hypothesis. A $\beta$ is generated from APP by concerted proteolysis by $\beta$-secretase, which generates carboxyl-terminal fragments (CTFs) of APP, and then by $\gamma$-secretase. The A $\beta$ forms aggregates in the extracellular compartment as senile plaques through a process that depends on proteoglycans and apolipoproteins. The extracellular $A \beta$ oligomers may activate caspases through activation of cell surface death receptors. Alternatively, intracellular A $\beta$ may activate caspases through a process that involves ER stress or mitochondrial stress. One of the consequences of caspase activation is cleavage of tau, which favors conformational changes characteristic of paired helical filaments (PHF-tau). Progressive accumulation of tau leads to cytoskeletal disruption (inset), failure of axoplasmic and dendritic transport, and subsequent loss of trophic support that culminates in neuronal death. The extracellular amyloid deposits in senile plaques also trigger reactive glial changes and neuroinflammation that can also contribute to neuronal loss through production of reactive oxygen species (ROS), NO, and proinflammatory cytokines such as TNF- $\alpha$ and IL-1 $\beta$. sAPP $\beta$, secreted APP $\beta$.

Enthusiasm for apoptosis, however, as a mechanism for neuronal death in AD has been tempered in recent years. The initial evidence for apoptosis in AD came from cell culture experiments that were not always physiologically relevant, including exposure of cells to very high concentrations of $A \beta$ or to $A \beta$ peptides that do not exist in nature. Evidence for frank cellular apoptosis in AD is controversial, but there is growing recognition that apoptotic mechanisms may play a role in disease pathogenesis in the absence of overt apoptosis (10). Apoptosis is an attractive mechanism for neuronal death in neurodegenerative diseases for several reasons. Neuronal death in degenerative diseases is selective at the individual cell level and not associated with inflammation. In $\mathrm{AD}$, neuronal loss is prominent in the cerebral cortex and the limbic lobe, while different neuronal populations are vulnerable in other neurodegenerative diseases. For example, in PD, brainstem monoaminergic neurons are affected. Cellular death via apoptosis is not associated with disruption of the cell membrane, and clearance of cellular debris is by facultative tissue phagocytes rather than professional macrophages of the reticuloendothelial system. Apoptosis also has a number of characteristic morphologic hallmarks, such as nuclear condensation and fragmentation, which have been very difficult to identify in $\mathrm{AD}$ brains.

Another feature of apoptosis is intranucleosomal cleavage that produces DNA nicks that can be labeled by specific techniques, such as TUNEL. Initial reports of extensive neuronal TUNEL labeling in $\mathrm{AD}$ have been difficult to confirm, and much of the labeling appears to be related to damage to DNA that occurs as a postmortem 


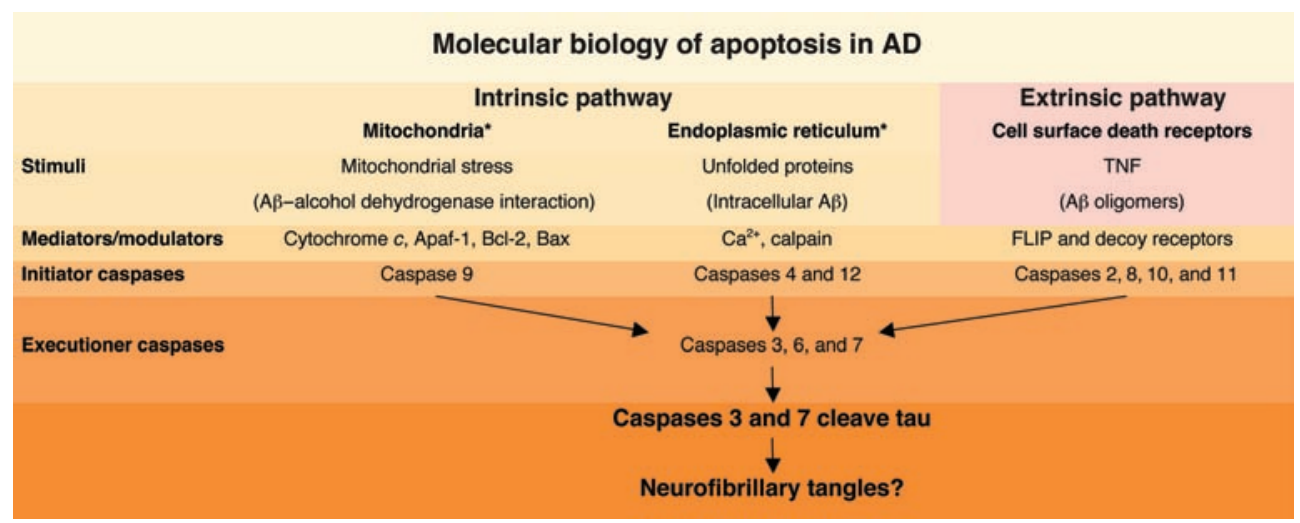

\section{Figure 2}

The interface of the two major molecules implicated in AD pathogenesis with molecular mechanisms of apoptosis. The two major pathways to cellular apoptosis are intrinsic and extrinsic. The extrinsic pathway involves signaling through cell surface death receptors, such as the TNF receptor, which are regulated by decoy receptors and Fas-associated death domain-like interleukin-1 $\beta$-converting enzyme inhibitory proteins (FLIPs). Direct binding of $A \beta$ or $A \beta$ oligomers to death receptors remains to be shown, but the pattern of activation of downstream caspases (e.g., caspases 2 and 8) supports involvement of the extrinsic pathway in A $\beta$-mediated apoptotic processes. Alternatively, intracellular $A \beta$ produced in the ER may lead to ER stress, or binding of $A \beta$ to a mitochondrial alcohol dehydrogenase may lead to mitochondrial stress. Both entries into the intrinsic pathway may activate downstream apoptotic mechanisms. While details of the upstream mechanisms and mediators remain to be defined, activated executioner caspases 3 and 7 are capable of cleaving tau protein, which may favor formation of NFTs. Asterisk indicates possible sites of action of $A \beta$. Caspases 1 and 5 are involved in cytokine activation.

artifact (11). Another approach to studying apoptosis is to examine enzymes that are involved in mediating programmed cell death. About a dozen such proteases have been identified; they share the property of being cysteine aspartyl proteases and are referred to as caspases. Caspases participate in apoptosis through initiation of intracellular cascades and in executing the final outcome, including proteolytic cleavage of cytoskeletal proteins and proteins of the nuclear scaffold. Both membrane (e.g., spectrin) and cytosolic (e.g., intermediate filament) cytoskeletal proteins are targets of caspase cleavage. Apoptosis is initiated through intracellular mechanisms that often involve alterations in mitochondria or endoplasmic reticulum and by signaling through cell membrane death receptors - the so-called intrinsic and extrinsic apoptotic pathways (12) (Figure 2).

Activation of caspases has been reported in $\mathrm{AD}$ using antibodies that are specific to the activated forms of the enzymes, which themselves are activated by proteolysis (13). Increasingly, apoptotic mechanisms are viewed to play a role in neurodegeneration in the absence of overt apoptosis. The process may even act in discrete subcellular domains such as synaptic termini (14). If caspase activation plays a role in $\mathrm{AD}$, it is important to learn how activation occurs, where in the neuron this process takes place, and whether or not it leads to neuronal death.

\section{Activation of apoptosis in AD}

While $A \beta$ is a leading candidate for activation of apoptotic mechanisms in $\mathrm{AD}$, recent evidence suggests that it is not the soluble form of $A \beta$ that has toxic properties, but rather, higher order complexes of $\mathrm{A} \beta$, including protofibrils and oligomers

(also referred to as $\mathrm{A} \beta$-derived diffusible ligands or ADDLs) (15). Some experimental studies suggest that $A \beta$ can activate caspases through the extrinsic pathway, implicating binding of extracellular A $\beta$ to cell receptors, while other studies suggest that the intrinsic pathway may

\section{Table 1}

Proteolysis in Alzheimer disease and Parkinson disease

$\begin{array}{lccc}\begin{array}{l}\text { Protein } \\ \text { APP }\end{array} & \text { Proteases } & \text { Proteolytic products } & \text { Disorder } \\ & \alpha \text {-Secretase } & \text { SAPP } \alpha & \text { Alzheimer disease } \\ & \beta \text {-Secretase } & \text { SAPP } \beta & \text { and aging } \\ & \alpha \text { - and } \gamma \text {-secretases } & \text { A } \beta 17-40 / 42(\mathrm{P} 3) & \\ & \beta \text { - and } \gamma \text {-secretases } & \text { A } \beta 1-40 / 42, A \beta 11-40 / 42 & \\ \text { Caspase 3 } & \text { C31 } & \text { Alzheimer disease } \\ \text { Tau } & \text { Caspases 3 and } 7 & \text { N-terminal fragment }(\Delta \text { tau }) & \text { and tauopathies } \\ & \text { Calpain } & \text { Multiple truncated species } & \text { Parkinson disease and } \\ \alpha \text {-Synuclein } & \text { Unknown } & \text { N- and C-terminal truncation } & \text { Lewy body dementia }\end{array}$

APP is cleaved by a number of different proteases to generate a range of peptide fragments that are found in the brain in AD and pathological aging. APP is cleaved by $\alpha$-secretase to generate secreted $\operatorname{APP} \alpha(\operatorname{sAPP} \alpha)$ and a carboxyl-terminal fragment. Further cleavage of this fragment by $\gamma$-secretase, generates $A \beta 17-40 / 42$, which is also referred to as P3. Secreted forms of APP have neurotrophic and neuroprotective properties. Similarly, $\beta$-secretase generates secreted APP $\beta$ (sAPP $\beta$ ) and a carboxyl-terminal fragment that upon further cleavage by $\gamma$-secretase generates $A \beta$. Heterogeneity of cleavage by both $\beta$-secretase and $\gamma$-secretase generates a family of peptides starting at residues 1 and 11 and ending at residues 40 and 42. APP is a target for caspase cleavage, which produces a carboxyl terminal 31 amino acid product as well. Tau cleavage by caspases 3 and 7 generates a protein truncated at residue Asp421 in the carboxyl half of the molecule, which has been referred to as $\Delta$ Tau. Calpains also cleave tau at a number of sites, depending on the enzyme concentration and the extent of proteolysis. Tau aggregates are characteristic not only of AD, but also of a family of neurodegenerative disorders referred to as the tauopathies. The protein that accumulates in Lewy bodies in Parkinson disease and dementia with Lewy bodies, $\alpha$-synuclein, is also subject to cleavage; however, the responsible proteases remain to be determined. 
be more relevant. Recent studies have drawn attention to the possible role of intracellular $A \beta$ in neurodegeneration (16, 17). Accumulation of $A \beta$ in endoplasmic reticulum or endosomes, where it may be synthesized, may activate apoptotic mechanisms through the unfolded protein response or endoplasmic reticulum stress. Alternatively, intracellular $A \beta$ may bind to alcohol dehydrogenase within mitochondria and activate apoptosis through mitochondrial stress (18).

One of the consequences of caspase activation as discussed by Rissman and coworkers (8), as well as in the previous studies by Gamblin and others (19), is cleavage of tau protein. This is a significant finding because, in order for tau to form fibrils similar to those in NFTs, a number of conditions must be met (20). Fragments of tau, particularly those that contain the microtubule-binding domain, which is critical for self-interaction, more readily aggregate into fibrils than full-length tau. Other substances contribute to efficient fibril formation, particularly polyanions such as heparan sulfate proteoglycans, RNA, or arachidonic acid.

\section{Tau proteolysis and neurofibrillary degeneration}

The role of conformational changes in tau is also considered by some to be critical (21). Since tau is a natively unfolded molecule, its crystal structure is unknown and most current ideas about conformational changes in tau are based upon speculation rather than any hard structural data. Conformational changes in tau are inferred from antibodies that react with tau in solution-based assays or with pathological tau in immunohistochemistry, but not with denatured tau in Western blots. The epitopes of these putative conformational antibodies may be discontinuous, and this is the case for the epitope recognized by $\operatorname{MC} 1(21,22)$, which is the monoclonal antibody used by Rissman and coworkers (8) to detect abnormal forms of tau protein. The recognition of this tau epitope by MC1 is dependent on specific amino- and carboxyl-terminal residues, which suggests that MC1 must recognize tau that is folded in such a way as to bring the two termini together (22). This conformation is felt to be specific for tau that forms NFTs (Figure 1 ). The conditions that favor this conformation are not known with certainty, but phosphorylation and proteolysis are postulated to be important. The evidence that phosphorylation is important is the fact that many of the antibodies that recognize NFTs recognize phosphoepitopes (23). Moreover, mapping of the phosphoepitopes in tau reveals phosphorylation sites that are not known to occur in normal tau. On the other hand, phosphorylation is not necessary for tau to form fibrils, at least in the test tube (20).

\section{Proteolysis in AD and other neurodegenerative disorders}

The first successful attempts to produce fibrils from tau used constructs with little more than the microtubule-binding domain, but more recent efforts show that full-length tau also forms fibrils in vitro. The microtubule-binding domain appears to be important for tau-tau interaction, and structural studies of the tau filaments isolated from Alzheimer brains reveal that the filaments have a fuzzy coat that can be cleared by protease treatment, leaving intact tau filaments. Analysis of the filaments with the fuzzy coat shows full-length tau, while the core contains the microtubule-binding domain of tau and a small number of flanking sequences (24).

If proteolytic events are crucial to the conformational changes that characterize early tau pathology, it is important to determine the proteases that catalyze this reaction. Tau undergoes proteolysis by at least two types of proteases - caspases and calpains $(25,26)$. Calpains are calcium-dependent proteases, and their activation by calcium fits well with pathogenetic scenarios that invoke increases in intracellular calcium as critical events in neurodegeneration. Caspases, on the other hand, are activated by conditions that favor apoptosis as discussed above. Interestingly, there is experimental evidence to suggest that both types of proteases can be activated during apoptosis (25). Moreover, proteolytic products generated by caspases and calpains can be difficult to differentiate from each other (27). While calpains are activated in nonapoptotic cell death, they may also play a role in the signal transduction that leads to apoptosis, particularly in the setting of endoplasmic reticulum stress.

Understanding the relationship of proteolysis of tau protein to subsequent conformational changes and fibrillar aggregation is likely to shed light not only on AD, but also on other common neurodegenerative disorders, including PD. In AD and PD there is compel- ling evidence that proteolytic processing events contribute to aggregation of abnormal proteins derived from normal cellular proteins (Table 1). Both APP and tau are subject to proteolysis in AD, which produces proteins with abnormal conformations that aggregate into fibrillar intracellular and extracellular lesions. Similarly, the protein that accumulates in Lewy bodies within degenerating monoaminergic neurons in PD, $\alpha$-synuclein, is also subject to proteolysis, since amino- and carboxyl-terminal truncated forms of $\alpha$-synuclein are enriched in diseased brains (28). As with $\mathrm{AD}$, it remains to be determined with certainty if the proteolytic events are necessary and early events that lead to neurodegeneration. Alternatively, they may be secondary processes in degenerating neurons subjected to activation of apoptotic mechanisms or flooding of intracellular compartments by calcium due to other underdetermined pathogenic processes.

Address correspondence to: Dennis W. Dickson, Department of Pathology (Neuropathology), Mayo Clinic, 4500 San Pablo Road, Jacksonville, Florida 32224, USA. Phone: (904) 953-7137; Fax: (904) 9537117; E-mail: dickson.dennis@mayo.edu.

1. Dickson, D.W. 1997. Pathogenesis of senile plaques. J. Neuropathol. Exp. Neurol. 56:321-339.

2. Grundke-Iqbal, I., et al. 1986. Microtubule-associated protein tau. A component of Alzheimer paired helical filaments. J. Biol. Chem. 261:6084-6089.

3. Tanzi, R.E., and Bertram, L. 2001. New frontiers in Alzheimer's disease genetics. Neuron. 32:181-184.

4. Grober, E., et al. 1999. Memory and mental status correlates of modified Braak staging. Neurobiol. Aging. 20:573-579.

5. Bennett, D.A., Schneider, J.A., Wilson, R.S., Bienias, J.L., and Arnold, S.E. 2004. Neurofibrillary tangles mediate the association of amyloid load with clinical Alzheimer disease and level of cognitive function. Arch. Neurol. 61:378-384.

6. Mandelkow, E.M., Stamer, K., Vogel, R., Thies, E., and Mandelkow, E. 2003. Clogging of axons by tau, inhibition of axonal traffic and starvation of synapses. Neurobiol. Aging. 24:1079-1085.

7. Golde, T.E. 2003. Alzheimer disease therapy: can the amyloid cascade be halted? J. Clin. Invest. 111:11-18. doi:10.1172/JCI200317527.

8. Rissman, R.A., et al. 2004. Caspase-cleavage of tau is an early event in Alzheimer disease tangle pathology. J. Clin. Invest. 114:121-130. doi:10.1172/ JCI200420640.

9. Cotman, C.W., and Anderson, A.J. 1995. A potential role for apoptosis in neurodegeneration and Alzheimer's disease. Mol. Neurobiol. 10:19-45.

10. Stadelmann, C., Bruck, W., Bancher, C., Jellinger, K., and Lassmann, H. 1998. Alzheimer disease: DNA fragmentation indicates increased neuronal vulnerability, but not apoptosis. J. Neuropathol. Exp. Neurol. 57:456-464.

11. Migheli, A., Cavalla, P., Marino, S., and Schiffer, D. 1994. A study of apoptosis in normal and pathologic nervous tissue after in situ end-labeling of 
DNA strand breaks. J. Neuropathol. Exp. Neurol. 53:606-616.

12. Schultz, D.R., and Harrington, W.J., Jr. 2003. Apoptosis: programmed cell death at a molecular level. Semin. Arthritis Rheum. 32:345-369.

13. Su, J.H., Zhao, M., Anderson, A.J., Srinivasan, A., and Cotman, C.W. 2001. Activated caspase-3 expression in Alzheimer's and aged control brain: correlation with Alzheimer pathology. Brain Res. 898:350-357.

14. Mattson, M.P., Keller, J.N., and Begley, J.G. 1998. Evidence for synaptic apoptosis. Exp. Neurol. 153:35-48.

15. Gong, Y., et al. 2003. Alzheimer's disease-affected brain: presence of oligomeric A beta ligands (ADDLs) suggests a molecular basis for reversible memory loss. Proc. Natl. Acad. Sci. U. S. A. 100:10417-10422.

16. Glabe, C. 2001. Intracellular mechanisms of amyloid accumulation and pathogenesis in Alzheimer's disease. J. Mol. Neurosci. 17:137-145.

17. Oddo, S., et al. 2003. Triple-transgenic model of Alzheimer's disease with plaques and tangles: intracellular Abeta and synaptic dysfunction. Neuron. 39:409-421.

18. Lustbader, J.W., et al. 2004. ABAD directly links Abeta to mitochondrial toxicity in Alzheimer's disease. Science. 304:448-452.

19. Gamblin, T.C., et al. 2003. Caspase cleavage of tau: linking amyloid and neurofibrillary tangles in Alzheimer's disease. Proc. Natl. Acad. Sci. U. S. A. 100:10032-10037.

20. Yen, S.H, Hutton, M., DeTure, M., Ko, L.W., and Nacharaju, P. 1999. Fibrillogenesis of tau: insights from tau missense mutations in FTDP-17. Brain Pathol. 9:695-705.

21. Weaver, C.L., Espinoza, M., Kress, Y., and Davies, P. 2000. Conformational change as one of the earliest alterations of tau in Alzheimer's disease. Neurobiol. Aging. 21:719-727.

22. Jicha, G.A., Bowser, R., Kazam, I.G., and Davies, P. 1997. Alz-50 and MC-1, a new monoclonal antibody raised to paired helical filaments, recognize conformational epitopes on recombinant tau. J. Neurosci. Res. 48:128-132.

23. Jicha, G.A., O'Donnell, A., Weaver, C., Angeletti, R., and Davies, P. 1999. Hierarchical phosphorylation of recombinant tau by the paired-helical filamentassociated protein kinase is dependent on cyclic AMP-dependent protein kinase. J. Neurochem. 72:214-224.

24. Wischik, C.M., et al. 1988. Isolation of a fragment of tau derived from the core of the paired helical filament of Alzheimer disease. Proc. Natl. Acad. Sci. U. S. A. 85:4506-4510.

25. Canu, N., et al. 1998. Tau cleavage and dephosphorylation in cerebellar granule neurons undergoing apoptosis. J. Neurosci. 18:7061-7074.

26. Johnson, G.V., Jope, R.S., and Binder, L.I. 1989. Proteolysis of tau by calpain. Biochem. Biophys. Res. Commun. 163:1505-1511.

27. Wang, K.K. 2000. Calpain and caspase: can you tell the difference? Trends Neurosci. 23:20-26.

28. Lee, M.K., et al. 2002. Human alpha-synucleinharboring familial Parkinson's disease-linked Ala-53 $\rightarrow$ Thr mutation causes neurodegenerative disease with alpha-synuclein aggregation in transgenic mice. Proc. Natl. Acad. Sci. U. S. A. 99:8968-8973. 